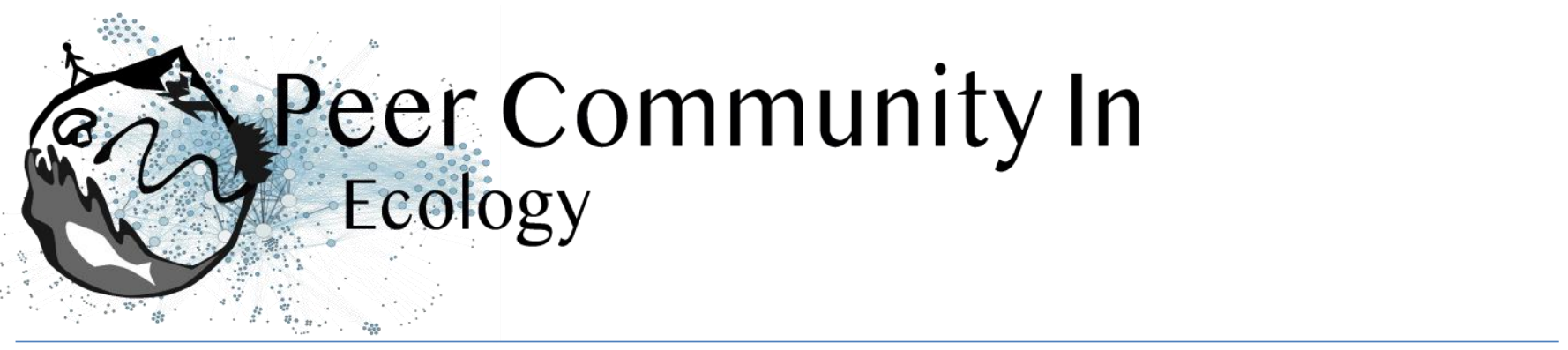

\title{
Allee effects under the magnifying glass
}

\author{
David Alonso based on reviews by Dani Oro, Tom Van Dooren and 1 \\ anonymous reviewer
}

\section{A recommendation of:}

The hidden side of the Allee effect: correlated demographic traits and extinction risk in experimental populations

Vercken Elodie, Groussier Géraldine, Lamy Laurent, Mailleret Ludovic(2021), HAL, hal02570868, ver. 4 peer-reviewed and recommended by Peer Community in Ecologyhttps://hal.archives-ouvertes.fr/hal-02570868

\section{Open Access}

Submitted: 30 September 2020, Recommended: 19 April 2021

\section{Recommendation}

Published: 22 April 2021

Copyright: This work is licensed under the Creative Commons Attribution-NoDerivatives 4.0 International License. To view a copy of this license, visit http://creativecommons.org/licen ses/by-nd/4.0/
For decades, the effect of population density on individual performance has been studied by ecologists using both theoretical, observational, and experimental approaches. The generally accepted definition of the Allee effect is a positive correlation between population density and average individual fitness that occurs at low population densities, while individual fitness is typically decreased through intraspecific competition for resources at high population densities. Allee effects are very relevant in conservation biology because species at low population densities would then be subjected to much higher extinction risks.

However, due to all kinds of stochasticity, low population numbers are always more vulnerable to extinction than larger population sizes. This effect by itself cannot be necessarily ascribed to lower individual performance at low densities, i.e, Allee effects. Vercken and colleagues (2021) address this challenging question and measure the extent to which average individual fitness is affected by population density analyzing 30 experimental populations. As a model system, they use populations of parasitoid wasps of the genus Trichogramma. They report Allee effect in 8 out 30 experimental populations. Vercken and colleagues's work has several strengths.

First of all, it is nice to see that they put theory at work. This is a very productive way of using theory in ecology. As a starting point, they look at what simple theoretical population models say about Allee effects (Lewis and Kareiva 1993; Amarasekare 1998; Boukal and Berec 2002). These models invariably predict a one-humped relation between population-density and per-capita growth rate. It is important to remark that pure logistic growth, the paradigm of density-dependence, would never predict such qualitative behavior. It is only when there is a depression of per-capita growth rates at low densities that true Allee effects arise. Second, these authors manage to not only experimentally test this main prediction but also report additional demographic traits that are consistently affected by population density. 


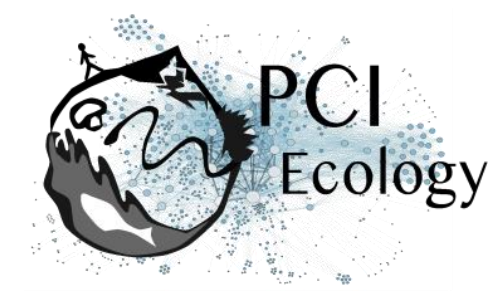

In these wasps, individual performance can be measured in terms of the average number of individuals every adult is able to put into the next generation ---the lambda parameter in their analysis. The first panel in figure 3 shows that the per-capita growth rates are lower in populations presenting Allee effects, the ones showing a one-humped behavior in the relation between per-capita growth rates and population densities (see figure 2). Also other population traits, such maximum population size and exitinction probability, change in a correlated and consistent manner.

In sum, Vercken and colleagues's results are experimentally solid and based on theory expectations. However, they are very intriguing. They find the signature of Allee effects in only 8 out 30 populations, all from the same genus Trichogramma, and some populations belonging to the same species (from different sampling sites) do not show consistently Allee effects. Where does this population variability comes from? What are the reasons underlying this within- and between-species variability? What are the individual mechanisms driving Allee effects in these populations? Good enough, this piece of work generates more intriguing questions than the question is able to clearly answer. Science is not a collection of final answers but instead good questions are the ones that make science progress.

\title{
References
}

Amarasekare P (1998) Allee Effects in Metapopulation Dynamics. The American Naturalist, 152, 298302. https://doi.org/10.1086/286169

Boukal DS, Berec L (2002) Single-species Models of the Allee Effect: Extinction Boundaries, Sex Ratios and Mate Encounters. Journal of Theoretical Biology, 218, 375-394. https://doi.org/10.1006/jtbi.2002.3084

Lewis MA, Kareiva P (1993) Allee Dynamics and the Spread of Invading Organisms. Theoretical Population Biology, 43, 141-158. https://doi.org/10.1006/tpbi.1993.1007

Vercken E, Groussier G, Lamy L, Mailleret L (2021) The hidden side of the Allee effect: correlated demographic traits and extinction risk in experimental populations. HAL, hal-02570868, ver. 4 peer-reviewed and recommended by Peer community in Ecology. https://hal.archives-ouvertes.fr/hal-02570868

Cite this recommendation as:

David Alonso (2021) Allee effects under the magnifying glass. Peer Community in Ecology, 100078. 10.24072/pci.ecology.100078

\section{Reviews}

Toggle reviews

\section{Revision round \#1}

\author{
2020-12-31
}

\section{Author's Reply}

Download author's reply (PDF file)Download tracked changes file

\section{Decision round \#1}

Dear Authors,

Your article submitted to $\mathrm{PCl}$ Ecology for evaluating a possible recommendation has now been reviewed by 3 referees and the referee reports can be found below and/or attached to this message.

Your paper has been found interesting but not good enough for a recommendation in its present form. Overall referees found your conclusions are not fully substantiated by the experiments you performed and the data you presented. For example, you clearly state in your abstract: "These results provide the first 
empirical demonstration of a correlation between the presence of positive density-dependence and impaired demographic performance, which increases the extinction risk of population, especially during the establishment phase". Although it is true that you find positive density-dependence in 3 (out of 20) populations, the statistical methods to come to your main conclusion need to be very much improved. Please follow the recommendations of van Dooren, who reviewed very thoroughly your statistical methods and did a great job at pointing to the main weaknesses in your analysis.

Notice that central figure 2 is not very conclusive. You need to add confidence bands to your plots as stated by referees 1 and 2. It might very misleading to add simply a curve as a guide to the eye as you did. For instance, panels pri1 and sem 4 could result from high stochasticity at low population numbers (as referee 1 mentions). Then you would be left only with panel cac_4, which seems clearer. Also, recall that figure 3 is based on the grouping sorting out presence and non-presence of Allee effects in your populations, but, if you are left only with one population (out of 20) showing significant Allee effects, then the statement "the presence of positive density-dependence and impaired demographic performance" (Fig 3) is clearly shaky.

My feeling is that you introduce with elegance the topic, but then you don't take advantage of your theoretical models in your statistical analyses. As a suggestion, you could use a time-discrete version of the modified logistic model of Lewis and Kareiva 1993, and then, in the light of the data you gathered, analyze the significance of the Allee effect parameters.

The discussion should be also rewritten in the light of referee recommendations, particularly, explaining (or speculating about) the mechanisms underlying the Allee effects seemingly found in your experimental populations. For instance, within the same species (cac), why do you find different results?

I believe your ms merits a recommendation only in case you are able to rewrite the paper strictly following referee recommendations. This may change some of your conclusions. You could even found kind of negative results, but this might be also interesting to report. You could be able to interpret your results differently, as referee 1 points out, in terms of stochasticity at low numbers. In sum, only if you are able to do a major and substantial revision, we could consider your ms suitable for a recommendation.

\section{David Alonso}

Referee 1

Download recommender's annotations (PDF)

Preprint DOI: https://hal.archives-ouvertes.fr/hal-02570868

\section{Reviewed by Dani Oro, 2020-10-20 17:11}

Download the review (PDF file)

\section{Reviewed by anonymous reviewer, 2020-11-25 11:54}

In this manuscript, the authors develop an experimental methodology to assess the presence of deterministic Allee effect in laboratory controlled growing populations of parasitoids. As stated by the authors, determining whether a population exhibit an Allee effect or not is correlated with an increased extinction risk at low population numbers. Determining whether Allee effects are present or not in growing populations has important implications for the development of populations and their potential survival. Given my area of expertise, I can evaluate the statistical methodology and the significance of the results that come out of the analysis. I cannot evaluate, however, the experimental design and the adequacy of the empirical assumptions used by the authors. Overall, I think that the manuscript is well written, concise and has a clear-cut objective. My global impression is that I can recommend this preprint to be published by PCI Ecology once a couple of questions about the statistical analyses are responded by the authors. 1. Fig. 2. Non-linear fits show clear evidence of Allee effect in three populations. However, there is a population (cac8) for which per-capita growth rate data seem to show an increase for small populations, but the presented fit does not seem to 
account for that fact (other populations like sem4 clearly account for that initial increase). Please, explain why and add a bit of discussion about this. 2. Given that experimental conditions are controlled and remain the same across populations, I find intriguing why all the cacoeciae populations do not exhibit Allee effects at all except for one population (cac_8). Do the authors have any idea of why this population is singular compared with the remaining cacoeciae? Please discuss this point a bit in the manuscript.

\section{Reviewed by Tom Van Dooren, 2020-11-20 17:01}

The preprint by Vercken et al presents results from an experiment where the growth rates of Trichogramma populations and their density-dependence were determined. The authors find three out of 20 cases where an Allee effect would be present.

I enjoyed reading the preprint but have doubts concerning the conclusions and would appreciate that the Allee-like effects discussed by e.g. Lande (1998) are treated from the introduction and more seriously considered. I would personally prefer reserving the term "Allee effects" for growth rate effects due to lack of social interactions at low densities. That does happen in the discussion but insufficiently so in general. In the introduction Allee effects are equated with positive density-dependence, which is a good choice as well, but then all potential mechanisms have to be introduced immediately. The abstract should define Allee effects as positive density dependence in that case as well. What you call "Allee-like" effects are there because of stochastic variability, interactions of means and variances. If you can attribute your results to them, please do so clearly. Lande (1998) calls them a type of Allee effect, so why not categorize different causes which can make one conclude from a time series analysis that there is positive density dependence and call all of them Allee effects?

I am unconvinced by the way Allee effects are detected: that happens just in the predicted time series, as if these predictions are perfect and without any uncertainty. You should consider the confidence bands for the predictions and detect an Allee effect from them.

Specific comments: Introduction: Plead add a citation to the first paper where the Allee effect was introduced. The papers added as references for the modified logistic model in fact refer to several different modified models, so there are "modified versions" of the logistic model. The terminology of Boukal and Berec is more precise than "weak" and "strong" Allee effects so please introduce it and use it. Maximum per capita growth rates are smaller with an Allee effect: does -A have to be smaller than $\mathrm{K}$ for this, please be more precise. Please state in the introduction how an Allee effect is detected in an actual population dynamical time series, so how its presence can be determined. Explain whether this then automatically implies that other statistics of the population dynamics will correlate with the strength of an Allee effect estimated from a time series or not. The introduction now gives us a feeling for what Allee effects do in models, but not how they emerge from time series analysis. I would already interpret a conclusion potentially differently here: did a time series with an extinction increase the likelihood of detecting an Allee effect of the kind Lande 1998 studied?

Methods: I don't agree with removing some of the time series from the analysis. You removed your true replicates. A mixed model analysis can deal with it easily: you did fit random line effects. There seemingly is an error in one of the sentences: one T. cordubensis population was removed for which there were several replicates? In the list the number (3) only goes down to (2). The text has to be written more clearly and state much earlier that replicates were initiated with different densities. You could drop the correlation tests on small population sizes, it's not treated in the results. The statistical analysis of per capita growth rate: Please use a Poisson model with an exponential link function. Your data are counts. Figure 2 suggest that your analysis might be too optimistic and underestimate expected demographic noise. I also don't see the need for the transformation in Eqn. 1. Use the density of the previous time step $t$ as an offset. Then the model fits In(lambda): in "R" a simple model version reads: $g \operatorname{lm}(\mathrm{N} t+1 \sim 1+\operatorname{offset}(\operatorname{In}(N \mathrm{t}))$, family = poisson). The intercept is $\ln ($ lambda). Fixing the spline parameter at $k=8$ in the presentation: first of all, which method was used to fit the models, and second, the figure doesn't give the impression $k$ was fixed. Was it allowed to be simplified automatically? Figure two needs to show confidence bands of the predictions. Glmm analysis: there are three 


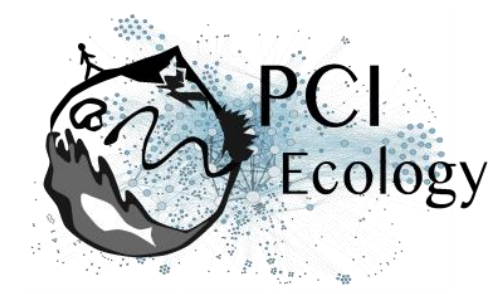

response variables. Three population sizes are converted into a maximum value and a mean, which is hardly any data reduction. The expected variability of the mean will depend on the length of the time series, so this should be accounted for. Since the response is multivariate, a multivariate model should be fitted with each replicate as unit of observation, and an overall anova test done of the Allee effect on these responses. In a way, however, this test is a bit strange, because the presence of an Allee effect is derived from grouped growth rates i.e. population density data (is this circular or not?) and as if the Allee effect is a lineage property which is perfectly known. However, you probably don't and you need to keep this in mind for the discussion. AIC are not good for hypothesis testing, because they tend to select models which are too rich in parameters (That's why they are very efficient at predicting). So simply use an anova applicable to your mixed models, which will probably be a parametric bootstrap for example. Interpreting the p-value of the fixed effect estimate is strange, because you already went through a frequentist procedure (the way you apply $\mathrm{AIC}$ ) before looking at these. In the equation for RI, what is "C_8"? If extinction occurs, isn't replicability automatically lower? Is it reasonable to use this measure, because when the difference is large after one generation, do you expect further large differences or again small ones? Do the densities at all time points contribute the same information? Maybe just $t=1$ is most informative?

Results: Figure two: connect the three points per replicate by a line. Confidence bands please for the predicted curves. Describe how you derive that an Allee effect is present from inspecting these confidence bands. If $\mathrm{k}$ changes automatically due to the model fitting procedure, indicate that. AIC: if you use AICc which is more pertinent, which values do you get? I find the p-values for the fixed effect parameters in two cases on the edge. What are the confidence intervals for these parameters? RI: what does a randomization test produce as $p$-value? The extinction probability of populations assigned to Allee is about $50 \%$. Maybe you are just detecting population extinctions with your Allee effect. What happens to the splines when you re-run the same three lineages, but without the replicates which went extinct?

Discussion: I cite from Lande 1998 on the Allee-like effect: ". For example, if the infinitesimal mean is positive and the infinitesimal variance increases with population size, it may nevertheless be true that starting from a given size most population trajectories tend to decrease, but that a small proportion of population trajectories undergo relatively large increases so that the expected population size increases with time" Please try to come up with a manner to falsify this hypothesis in your data. I believe you should give more attention to variability in the amounts of stochasticity between lineages, not just by calculating the RI index. The discussion, second paragraph, already suggest that differences in the amounts of stochasticity determine what you observed. If they do, you actually did a study detecting stochastic aspects of population dynamics. Please make it very clear what the difference is between weak Allee effects and stochastics producing the same patterns as Allee-effects (here they seem to be defined as effects due to the lack of interactions again). 